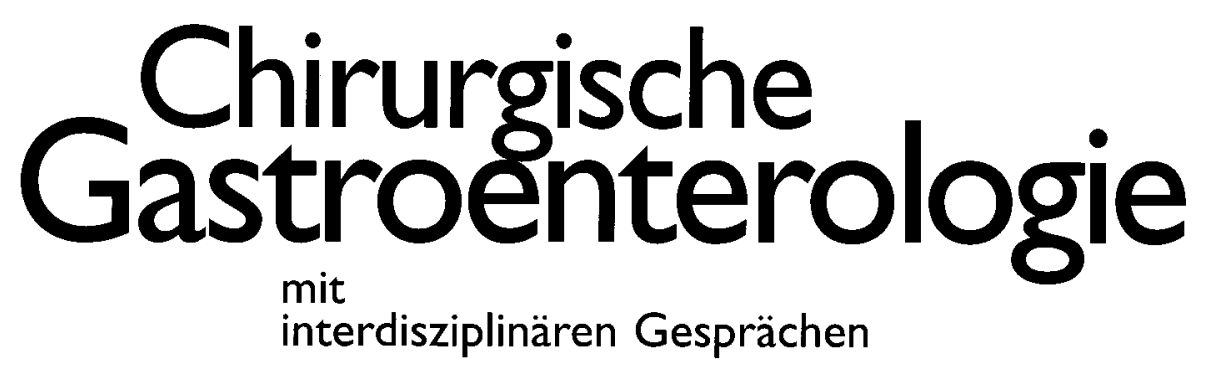

Hauptthema

G. Jatzko,

St. Veit/Glan

R. Bittner,

Stuttgart (Hrsg.)

\title{
Chirurgische Therapie des Magenkarzinoms
}

Eingeladene Beiträge

Weitere Rubriken

Originalarbeiten

Prä- und postoperative Therapie

Buchbesprechungen

Tagungen und Kongresse 


\section{Gründungsherausgeber}

A. Akovbiantz $\dagger$, Zürich

H. Denck, Wien

K.-J. Paquet, Bad Kissingen

C. E. Zöckler, Bad Oeynhausen

\section{Hauptschriftleiter}

R. Bittner, Stuttgart

K.-J. Paquet, Hannover

\section{Schriftleitung}

C. E. Broelsch, Essen

M. W. Büchler, Bern

I. Gastinger, Cottbus

K. Glaser, Wien

J. Horn, München

J. R. Izbicki, Hamburg

E. Klar, Heidelberg

J. Mössner, Leipzig

H. Säuberli, Baden

R. Schiessel, Wien

W. G. Zoller, Stuttgart

\section{Wissenschaftlicher Beirat}

J. Alexander-Williams, Birmingham

H. M. Becker, München

M. Betzler, Essen

E. Bodner, Innsbruck

H. Bosseckert, Jena

H. Bülow, Schweinfurt

R. Dölp, Fulda

K. E. Frede, Basel

J. Gallinger, Moskau

A. Gangl, Wien

J. M. Hackl, Innsbruck

E. G. Hahn, Erlangen/Nürnberg

J. Hauss, Leipzig

J. M. Henderson, Cleveland

P. Hermanek, Erlangen/Nürnberg

R. A. Hinder, Omaha

W. Hohenberger, Erlangen/Nürnberg

Y. Idezuki, Tokyo

I. Ihse, Lund

C. W. Imrie, Glasgow

G. G. Jamieson, Adelaide

S. L. Jensen, Århus

K. Junghanns, Ludwigsburg

P. J. Kestens, Brüssel

B. Langer, Toronto

S. Liebe, Rostock

H. Lippert, Magdeburg
P. Malfertheiner, Magdeburg

K. Maruyama, Tokyo

C. Meyer, Straßburg

W. C. Meyers, Worcester

P. Morel, Genf

E. Moreno-Gonzáles, Madrid

V. Pegan, Ljubljana

F. M. Penninckx, Leuven

A. Peracchia, Mailand

R. Porschen, Tübingen

H. B. Reith, Würzburg

J. F. Riemann, Ludwigshafen

L. F. Rikkers, Madison

J. Rodés, Barcelona

H.-D. Saeger, Dresden

R. Schlumpf, Aarau

W.-H. Schmiegel, Bochum

J. Schölmerich, Regensburg

M. Stolte, Bayreuth

K. Sugimachi, Fukuoka

J. Terblanche, Kapstadt

M. Thelen, Mainz

A. Thiede, Würzburg

A. L. Warshaw, Boston

C. Wittekind, Leipzig

Z. Wu, Wuhan

\section{Bibliographische Dienste:}

EMBASE / Excerpta Medica

Reference Update

S. Karger $\cdot$ Medical and Scientific Publishers $\cdot$ Basel $\cdot$ Freiburg $\cdot$ Paris $\cdot$ London $\cdot$ New York $\cdot$ New Delhi $\cdot$ Bangkok $\cdot$ Singapore $\cdot$ Tokyo $\cdot$ Sydney

Die Zeitschrift erscheint dreimonatlich: pro Jahr erscheint 1 Band zu je 4 Heften. Bezugspreis für Jahrgang 15, 1999 DEM 220.- / SFr 172,- einschließlich MwSt., zuzüglich Postgebühren. Der Abonnementpreis ist im voraus zahlbar. Da Abonnement der Zeitschrift läuft weiter, wenn es nicht spätestens 4 Wochen vor Abschluß eines Bandes abbestellt wird.

Abonnementbestellungen können bei jeder Buchhandlung oder direkt beim Verlag aufgegeben werden:

S. Karger GmbH

Lörracher Str. 16 a

D-79115 Freiburg

Telefon +49761452070

Telefax +497614520714

Übrige Länder:

S. Karger AG

Allschwilerstr. 10

Postfach

CH-4009 Basel

Telefon +41613061111

E-mail kargergmbh@aol.com Telefax +41613061234

Postbank München 61440-808 E-mail Karger@Karger.ch
Anzeigen: S. Karger

Verlag für Medizin und Naturwissenschaften $\mathrm{GmbH}$

Lörracher Str. 16 a. D-79115 Freiburg, Tel. +49 761452070

Gültig ist die Preisliste Nr. 7 vom 1. Januar 1999

Für den Inhalt außerhalb des redaktionellen Teils (insbesondere Anzeigen, Industrieinformationen, Pressezitate und Kongreßinformationen) übernehmen Schriftleitung, Beirat und Verlag keine Gewähr.

Eine Markenbezeichnung kann warenzeichenrechtlich geschützt sein, auch wenn bei ihrer Verwendung in dieser Zeitschrift das Zeichen ${ }^{\circledR}$ oder ein anderer Hinweis auf etwa bestehende Schutzrechte fehlen sollte. Für Satzfehler, insbesondere bei Dosierungsangaben, wird keine Gewähr übernommen.

Die Zeitschrift sowie alle in ihr enthaltenen einzelnen Beiträge und Abbildungen sind urheberrechtlich geschützt. Jede Verwertung, die nicht ausdrücklich vom Urheberrechtsgesetz zu- gelassen ist, bedarf der vorherigen Zustimmung des Verlags. Das gilt insbesondere für Vervielfältigung, Bearbeitungen, Übersetzungen, Mikroverfilmungen und die Einspeicherung und Verarbeitung in elektronischen Systemen.

(c) Copyright 1999 by S. Karger

Verlag für Medizin und Naturwissenschaften $\mathrm{GmbH}$ Lörracher Straße 16 a, D-79115 Freiburg

Verlagsleitung und presserechtlich verantwortlich: Sibylle Hopf

Redaktionsassistenz: Dr. Hannelore Tenckhoff

Anzeigenleitung: Susanne Meister

Produktionsleitung: Georg Brunner

Herstellung: druckhaus köthen $\mathrm{GmbH}$

Friedrichstraße 11/12, D-06366 Köthen/Anhalt

ISBN 3-8055-6831-2

\section{KARGER}

Fax +497614520714

E-mailkargergmbh@aol.com

www.karger.com 
Editorial

210 Chirurgische Therapie des Magenkarzinoms Jatzko, G. (St. Veit/Glan); Bittner, R. (Stuttgart)

Hauptthema

211 Magenkarzinom: Epidemiologie, Risikofaktoren und Rolle der Ernährung

Püspök, A.; Ferenci, P. (Wien)

216 Pathologie des Magenkarzinoms: Typing, Staging und Grading

Wittekind, C. (Leipzig)

223 Chirurgische Therapie und Ergebnisse beim Magenfrühkarzinom

Jatzko, G.; Pertl, A.; Jagoditsch, M. (St. Veit/Glan)

227 Standardresektion und Lymphadenektomie beim Magenkarzinom

Lehnert, T.; Kienle, P.; Maksan, S.; Herfarth, C. (Heidelberg)

237 Chirurgische Therapie bei Karzinomen des proximalen Magendrittels und des ösophagogastralen Übergangs Böttcher, K.; Etter, M.; Stein, H. J.; Roder, J. D.; Siewert, J. R. (München)

244 D2- versus D1-Lymphknotendissektion: Ergebnisse der Medical-Research-Council(MRC)- und der niederländischen Studie (Dutch Trial)

Whiting, J. L.; Fielding, J. W. L. (Birmingham)

249 Stadienverschiebung («stage migration») beim Magenkarzinom: Einfluß auf die Überlebensrate Hermans, J.; Bonenkamp, J. J.; Sasako, M.; van de Velde, C. J. H. (Leiden)

253 Ösophagojejunostomie: Mechanische Klammernaht versus Handnaht Seufert, R. M. (Remscheid)
Editorial

210 Surgical Therapy of Gastric Cancer

Jatzko, G. (St. Veit/Glan); Bittner, R. (Stuttgart)

Main Theme

211 Gastric Cancer: Epidemiology, Risk Factors and Significance of Nutrition

Püspök, A.; Ferenci, P. (Wien)

216 Pathology of Gastric Cancer: Typing, Staging and Grading

Wittekind, C. (Leipzig)

223 Surgical Therapy and Results in Patients with Early Gastric Cancer

Jatzko, G.; Pertl, A.; Jagoditsch, M. (St. Veit/Glan)

227 Standard Resection and Lymhadenectomy in Gastric Cancer

Lehnert, T.; Kienle, P.; Maksan, S.; Herfarth, C. (Heidelberg)

237 Surgical Therapy of Proximal-Third Gastric Cancer and Adenocarcinoma of the Esophagogastric Junction Böttcher, K.; Etter, M.; Stein, H. J.; Roder, J. D.; Siewert, J. R. (München)

244 D2 versus D1 Lymph Node Dissection: Results of the Medical Research Council and the Dutch Trial

Whiting, J. L.; Fielding, J. W. L. (Birmingham)

249 Stage Migration in Gastric Cancer: Its Influence on Survival Rates

Hermans, J.; Bonenkamp, J. J.; Sasako, M.; van de Velde, C. J. H. (Leiden)

253 Esophagojejunostomy: Mechanically Stapled versus HandSutured Anastomoses Seufert, R. M. (Remscheid)

\section{KARGER}

Fax +497614520714

E-mail kargergmbh@aol.com

www.karger.com
(C) 1999 S. Karger GmbH, Freiburg

Artikel (Volltext) und Inhaltsverzeichnisse sowie das vorläufige Inhaltsverzeichnis des nächsten Heftes: www.karger.com/journals/cga/cga_bk.htm 
260 Aktueller Stand der laparoskopischen Chirurgie des Magenkarzinoms

Bärlehner, E. (Berlin)

Originalarbeiten

266 Antibiotikaprophylaxe in der Gallenwegchirurgie Wittmann, D. H. (Brookfield, WI)

274 Hepatikolithiasis - endoskopisch-interventionelle Therapiemöglichkeiten

Jakobs, R.; Riemann, J. F. (Ludwigshafen)

280 Primär sklerosierende Cholangitis - Therapieoptionen Maier, K. P. (Esslingen)

286 Indikationen und Ergebnisse der Lebertransplantation bei primär sklerosierender Cholangitis

Otto, G. (Mainz)

Prä- und postoperative Therapie

292 Interdisziplinäres Gespräch: Neoadjuvante und adjuvante Therapie beim Ösophaguskarzinom

Petrasch, S. (Bottrop) (Gesprächsleiter)

300 Buchbesprechung

303 Tagungen und Kongresse

206 Impressum

U3 Richtlinien für Autoren
260 Laparoscopic Gastric Surgery for Carcinoma: State of the Art

Bärlehner, E. (Berlin)

Original Articles

266 Antibiotic Prophyaxis in Bile Duct Surgery Wittmann, D. H (Brookfield, WI)

274 Hepatic Lithiasis - Endoscopic Treatment Options

Jakobs, R.; Riemann, J. F. (Ludwigshafen)

280 Treatment of Primary Sclerosing Cholangitis Maier, K. P. (Esslingen)

286 Indications and Results of Liver Transplantation in Primary Sclerosing Cholangitis Otto, G. (Mainz)

Pre- and Postoperative Therapy

292 Interdisciplinary Discussion: Neoadjuvant and Adjuvant Therapy in Patients with Esophageal Cancer Petrasch, S. (Bottrop) (Discussion Leader)

300 Book Reviews

303 Meetings and Conferences

206 Imprint

304 Guidelines for Authors

\section{KARGER}

Fax +497614520714

E-mailkargergmbh@aol.com

www.karger.com (c) 1999 S. Karger GmbH, Freiburg

Artikel (Volltext) und Inhaltsverzeichnisse sowie das vorläufige Inhaltsverzeichnis des nächsten Heftes: www.karger.com/journals/cga/cga_bk.htm 


\section{In Vorbereitung}

Leiomyosarkom der Leber und Vena-cava-Kompressionssyndrom bei einer 66jährigen Patientin - eine Kasuistik

Kahlenberg, D.; Will, U.; Scheele, J.; Werner, B.; Katenkamp, D.; Bosseckert, H. (Jena)

Subklinische portosystemische Enzephalopathie und Lungenstrombahnveränderungen bei Kindern und Jugendlichen mit portaler Hypertension bei prähepatischem Block Otting, U.; Hellmann, C.; Planke, C.; Lehmann, R.; Gottschalk, S. (Berlin)

Endoskopische Behandlung der Subtypen des frühen Magenkarzinoms

Shimao, H.; Hiki, Y.; Morise, M.; Kikuchi, S.; Kobayashi, N.; Sakakibara, Y.; Kakita, A.; Tanabe, S.; Saigenji, K. (Sagamihara); Tsutsumi, O. (Braunschweig)

Zystenkrankheit des Ductus Choledochus und Karzinomrisiko - ein ungewöhnlicher Verlauf

Gottschalk, U.; Boden, G. (Berlin)

\section{KARGER}

Fax +497614520714

E-mailkargergmbh@aol.com

www.karger.com 